\title{
Viviendo con una enfermedad crónica fatal: significados en una muestra brasileña*
}

\section{Living with a Fatal Chronic Disease: Meanings in a Brazilian Sample}

Recibido: mayo 15 de 2010 | Revisado: julio 26 de 2011 | Aceptado: junio 20 de 2012

\author{
KELLY SiMÕES ** \\ EDSON SOUZA FILHO *** \\ Universidade Federal do Rio de Janeiro, Brasil
}

\begin{abstract}
RES UMEN
La enfermedad crónica fatal impacta la vida con transformaciones en términos conductuales y afectivos. Los objetivos de este trabajo fueron: identificar el significado de la vivencia de la enfermedad crónica fatal y percibir el significado del concepto de salud y enfermedad, en una muestra brasileña de portadores de enfermedad crónica fatal. Para esto, se adoptó una posición teórica psicosocial de análisis subjetivo, según el abordaje de las representaciones sociales. En cuanto al método, la investigación fue cualitativa y utilizó estudios de caso en pacientes internados portadores de enfermedades crónicas fatales. Se concluyó que es la enfermedad en sí misma lo que parece ocupar un lugar central y dar sentido a los comportamientos y movilizaciones, y que el concepto de salud queda supeditado al estado de enfermedad.

Palabras clave autores

Enfermedad crónica, vida, salud.

Palabras clave descriptores

Psicología de la Salud, calidad de vida, investigación cualitativa.
\end{abstract}

\begin{abstract}
A B S T R A C T the state of the disease.

Key words authors

Chronic illness, life, health.

Key words plus

Health Psychology, Quality of Life, Qualitative Research.
\end{abstract}

The fatal chronic disease brings impacts to life with transformations in behavioural and affectionate terms. The objectives of this work were to identify the meaning of living the fatal chronic disease and to realise its meaning about of health and disease in Brazilian simple from the bearess of the disease. Thus, we adopted a psychosocial theoretical position for the subjectivity analysis, according to a social representations approach. In relation to the method, the research was qualitative, exploratory and it used studies of the cases among patients disease in the hospital. We concluded that it is the disease in itself which seems to occupy a central place and give sense to behaviours and mobilizations, the concept of health is attached to 


\section{Introducción}

El proceso de vida ocurre en el cotidiano de cada universo personal y social al mismo tiempo y conlleva una configuración singular de acuerdo con la historia, los valores y el momento presente. Así, se puede observar cómo este movimiento personal en el proceso de vida se configura con la presencia de una variable: la enfermedad crónica.

La incorporación de la enfermedad en la vida puede ser percibida como una transformación. El paciente atraviesa una transición que implica un cambio significativo de valores, identidad y actividades que pueden ser descritos como más importantes que retornar al estado normal (Beltrán-Salazar, 2008; Robins, 2005 citado en Russell \& Seeley, 2010; Zapata, 2011).

Según datos de la Organización Mundial de la Salud ([OMS], 2010, p. 71), dentro de algunos años y décadas el manejo de las condiciones crónicas será uno de los mayores desafíos que encararán los sistemas de salud en el mundo. En ese sentido, dicha organización (OMS, 2003) llama la atención sobre el hecho de que la frustración de las preferencias de los pacientes en lo que respecta a las decisiones implicadas en el tratamiento, no sean tenidas en consideración. Esto tiene que ver con que los pacientes se sientan menos facultados y sigan presentando actitudes negativas en relación con las decisiones en el tratamiento.

Frostholm et al. (2005) señalan cuestiones relevantes, como las percepciones del paciente acerca de la enfermedad, como guía en las decisiones del médico, lo cual tiene implicaciones si se considera al paciente como un experto. Declaraciones explícitas sobre sus ideas y conceptos acerca de la enfermedad pueden ampliar el espacio para que modelos biopsicosociales se tornen prácticos, con la expectativa de que los pacientes se beneficien con la reducción del riesgo de desarrollar condiciones crónicas, cuando podrían ser evitadas o aliviadas.

Por lo tanto, como en cualquier enfermedad que conlleva amenazas a la vida, en especial en el VIH/ SIDA, se debe encaminar a los pacientes para que se sientan más cómodos con los sistemas de apoyo; se debe estar atento a potenciales perfiles de depresión o suicidio y al impacto en el ámbito del trabajo, las finanzas y los sistemas de salud.

La experiencia subjetiva de los hechos de salud y enfermedad ha sido estudiada en los últimos años (Flick, 1993). Se trata de considerar acciones y evaluaciones de estados, interiores y exteriores, consigo mismo y en sus relaciones con el mundo social y objetivo. La posición teórica de este trabajo parte del supuesto de que la experiencia individual en eventos de salud/enfermedad es afectada por el sentido común, tal como este es reelaborado a través de las representaciones sociales (Moscovici, 1961/1976), de la misma manera como la ciencia y sus prácticas también se reconstruyen mediante el mismo fenómeno de conocimiento popular (Moscovici \& Hewstone, 1992).

Según la teoría mencionada, el hombre moderno participa activamente en la construcción social de la realidad objectiva y subjectiva, a partir de representaciones sociales, sea como individuo enfermo que se confronta con la enfermedad y con diferentes medios sociales, sea como grupo de legos en asuntos de salud/enfermedad, que se representan diferentes cuadros de enfermedad y de salud. Estos procesos ocurren según dos fenómenos relevantes: el anclaje de la experiencia, durante el cual la situación es transformada según marcos de referencia psicosocioculturales -por medio de significados, metáforas, conceptos y reglas sociales que están asociados con ellos-, y el uso práctico cotidiano refrendado.

Así pues, en términos de información, la búsqueda de regularidades es, en general, una intención del sujeto cognitivo, lo que permitiría no solamente validar el conocimiento en sí, sino también, en muchos casos, corresponder a expectativas sociales de identificación con el grupo, aunque otras funciones pueden estar en proceso de búsqueda tanto por parte del individuo enfermo como por el grupo al cual pertenece, como por ejemplo anticipar o justificar acciones (p. ej., Abric, 1994). La objectivación es el otro proceso importante de la construcción de la representación social, que es la transformación del evento en algo visible y concreto, lo que facilita o permite la evaluación del fenómeno en sí y, en consecuencia, perfecciona la comunicación social, sea 
con otros individuos del propio medio social de los sujetos enfermos, sea con miembros de otros grupos, como los profesionales de la salud.

En un mundo donde cada vez más hay acceso a la información y todos son llamados a decidir sobre lo que se debe hacer al respecto de la salud y el tratamiento de las enfermedades, este proceso empieza a partir de la selección de elementos del problema (enfermedad y enfermo), según los criterios valorativos del sujeto; pasa por el desplazamiento del contexto de conocimiento originalmente situado fuera de sí mismo (el campo médico o el grupo de pertenencia previo del sujeto individual) hacia el espacio privado y libre del sujeto individual o grupal y culminando, muchas veces, con la conformación de un soporte o núcleo figurativo del asunto.

Por lo tanto, otros criterios no necesariamente "racionales" interfieren en la experiencia humana de conocimiento y vivencia de la enfermedad, como la incertidumbre sobre el pronóstico de salud/enfermedad que constituye una información útil para sí mismo y para su entorno social (Giraldo, 2008), lo que puede generar sufrimiento, desgaste psicológico y consecuente fragilización del enfermo. Esta situación puede intensificarse cuando también produce incertidumbre en el entorno social más acostumbrado a cuadros de enfermedades considerados como eventos separados de la vida cotidiana, ya que esta última se caracteriza por ser vivida en estado de salud. El prolongamiento de la enfermedad sin pronóstico definido puede provocar sentimientos de soledad en el enfermo, debido a que no corresponde a la expectativa de identificación social.

Por último, se parte del supuesto de que esta dimensión subjetiva interfiere directamente sobre la evolución de muchos cuadros clínicos. En ese sentido, un estudio realizado en New York con portadores del virus del SIDA, mostró que quienes evaluaban sus vidas como contando con apoyo social, tendían a retardar significativamente el posterior desarrollo de síntomas de la enfermedad (Zich \& Temoshok, 1987).

Así, se analiza la forma en que las personas portadoras de enfermedades crónicas viven y perciben su propia salud y calidad de vida en ese día a día tan sufrido y lleno de obstáculos. En este contex- to, la presente investigación tuvo como objetivos: identificar la vivencia de la enfermedad crónica fatal y percibir el significado del concepto de salud y enfermedad en una muestra brasileña.

\section{Método}

Se trata de una investigación cualitativa en la que se utilizó la metodología de estudio de caso para alcanzar los objetivos propuestos.

\section{Participantes}

El presente proyecto fue desarrollado con personas portadoras de enfermedades crónicas fatales. La muestra estudiada en los estudios de caso estuvo compuesta por 10 pacientes con el perfil elegido, es decir, portadores de enfermedad crónica fatal en tratamiento, relativo a las siguientes enfermedades crónicas: SIDA (2 casos), cáncer (2 casos), cirrosis hepática (2 casos), EPOC (1 caso), Lupus Eritematoso Sistémico (1 caso), Insuficiencia Renal Crónica (2 casos). El promedio de edad fue de 58.3 años, y la muestra fue equilibrada en términos de género, escolaridad y aspectos socioeconómicos.

El grupo fue seleccionado aleatoriamente en los hospitales, teniendo como criterio de inclusión ser mayor de 18 años, mostrarse lúcido y orientado, poseer una enfermedad crónica fatal en tratamiento, estar internado en enfermerías debido al agravamiento o inestabilidad del cuadro clínico y en acompañamiento ambulatorio anterior al ingreso. Todos los participantes que aceptaron hacer parte de la investigación firmaron el consentimiento libre, previo e informado, aprobado por el Comité de Ética e Investigación.

Instrumento de recolección y análisis de datos

Dado que la investigación fue cualitativa, se empleó como instrumento una entrevista semiestructurada con preguntas abiertas que pretendía determinar los significados atribuidos por las personas portadoras de enfermedades crónicas a su proceso de vivir. La entrevista presentaba en su desarrollo preguntas que se referían al descubrimiento de la enfermedad 
y la trayectoria recorrida hasta los días actuales: icómo es vivir con determinada enfermedad?, icuál es la relación que se establece entre salud-enfermedad?

Se analizaron los datos teniendo en cuenta los significados explicitados en las palabras, procurando focalizar los aspectos psicológicos relacionados con el enfrentamiento de la enfermedad y cuestiones acerca de salud y enfermedad, con el fin de describir lo que tienen en común los varios cuadros clínicos en portadores de una enfermedad crónica fatal, es decir, que evolucionará hasta la muerte. La diversidad de las enfermedades no impidió el análisis, sino que más bien posibilitó la oportunidad de una descripción más general.

Luego de la realización de las entrevistas semiestructuradas con personas portadoras de enfermedades crónicas en fase crónica del intervalo vivir-morir, los datos fueron analizados con la técnica de Análisis de Contenido (Bardin, 1993).

Se pueden observar a continuación las categorías que surgieron en el discurso de los pacientes, con sus ejemplos respectivos.

\section{Temática 1: Involucramiento con la enfermedad}

\section{Vive en función de la enfermedad}

La experiencia de la enfermedad es modulada por el diálogo y el conflicto entre las representaciones sociales y las que el individuo enfermo tiene de sí mismo.

Ejemplo:

(...) las personas que no entienden también te ven saludable aparentemente ahí piensan que estás de broma que eres perezoso que no quieres nada, ientendiste? y la persona está allá enferma (...) la falta de libertad de alimentación tampoco todo puede ser comido... (Entrevistado 5)

\section{La enfermedad como parte de la vida}

La experiencia de la enfermedad es aceptada como un hecho médico y social, frente al cual el individuo enfermo se siente asociado en corresponsabilidad.
Ejemplo:

(...) ya tenía medicamento para esas cosas, entonces yo... fui llevando... y por un lado me quedé tranquilo... (Entrevistado 2)

\section{Temática 2: Relación salud-enfermedad}

\section{La enfermedad como pérdida de la salud}

La experiencia de la enfermedad es representada como pérdida de algo considerado muy importante e insustituible para que el individuo pueda vivir: la salud.

Ejemplo:

(...) pero las personas enfermas nada son... la persona enferma no es nada de veras... (Entrevistado 6)

Momento saludable X Momento enfermo

La experiencia de la enfermedad es representada como resultado de contraposiciones entre tiempos regulares y repetidos en que se goza de buena salud, seguidos por periodos en que se está enfermo.

Ejemplo:

(...) Entonces uno se queda débil... ile da flojera? sí... dolor también... creo que es dolor... es... pero cuando uno está bien, es prácticamente normal... tomando medicamento a diario... correctamente... normal... (Entrevistado 2)

\section{Resultados y discusión}

A partir de lo expuesto en el método, se describe enseguida la caracterización de la muestra estudiada, teniendo en cuenta los aspectos sociodemográficos y las categorías temáticas creadas a partir del análisis de contenido.

En lo relativo a los aspectos sociodemográficos de la muestra de portadores de enfermedad crónica fatal, en cuanto a la edad se encontró: 1 participante en la franja etaria de 18 a 30 años; 4, entre 41 y 50 años y 4 , entre 51 y 60 años; en cuanto al sexo, 4 mujeres y 6 hombres, y en relación con la escolaridad, 2 con educación elemental incompleta, 5 con 
educación elemental completa, 1 con educación secundaria incompleta y 2 con educación secundaria completa.

De acuerdo a los resultados obtenidos y presentados, fruto del análisis de contenido de las entrevistas realizadas, los datos se discuten a la luz de la literatura especializada a fin de establecer paralelos y ampliar el debate respecto del fenómeno estudiado a través de los casos recolectados: la vivencia de la enfermedad crónica fatal.

\section{Involucramiento con la enfermedad}

En cuanto al involucramiento con la enfermedad, las personas portadoras de enfermedades crónicas fatales refirieron que viven en función de la enfermedad.

Es una renuncia de todo que yo ya hice en mi vida... yo... yo... tengo cabeza... siempre tuve una cabeza muy buena pero cuando la persona queda en este estado, usted solo se deprime se cierra así en la cáscara del huevo y va muriendo poco a poco, no quiere más saber de vida y voy a decirte más, tú crees que a veces yo me levanto por la mañana y no quiero comer, no quiero bañarme, no quiero cambiar de ropa, yo me levanto... mi hijo hizo una mesa de madera en el quintal que yo le pedí a él con banco así con todo allí, queda todo: ventilador, televisión, almohada, nebulizador, iqueda todo! (Entrevistado 7)

En la enfermedad crónica fatal, la vida de la persona portadora se desarrolla en función de la enfermedad. La enfermedad abarca y domina a quien es portador; se hace difícil vivir sin sufrir las consecuencias que trae consigo, ya sean físicas, emocionales, sociales o económicas.

Bapat, Kedlaya y Gokulnath (2009) afirman que la enfermedad llega como una intrusa con mayores o menores extensiones, resultando muy afectadas áreas como el trabajo, la salud, las finanzas, las dietas, la vida sexual y el estado psicológico.

De esta forma, es posible pensar que la presencia de la enfermedad crónica se hace protuberante y parece adquirir una centralidad en el vivir de sus portadores; trae desafíos que dificultan el transcu- rrir de esta experiencia sin daño. Ciertamente, el enfermar no se instaura como un momento, sino como un proceso que avanza día a día: vida y enfermedad.

Jeon, Kraus, Jowsey y Glasgow (2010) afirmaron que con frecuencia los pacientes encuentran dificultades en lo que se refiere a la realización de los ajustes necesarios para adaptarse. Ser flexible para modificar circunstancias es una cuestión que exige que el paciente sea consciente, acepte las limitaciones físicas y situaciones de la nueva vida y se adapte de la mejor forma posible (Beltrán-Salazar, 2010).

Flume et al. (2010) llaman la atención, en el caso de pacientes con fibrosis cística, al hecho de que se pueden encontrar las necesidades específicas tales como son determinadas por estos individuos, sus familias y recomendadas por cuidadores en los equipos de salud, ya que los protocolos deben facilitar el tratamiento, mejorar o extender sus vidas.

Así, estos pacientes demandan un proceso activo de gestión que va más allá de la perspectiva técnica y médica, pues implica a todas las esferas de su vida social. Las personas afectadas necesitan lograr cambios profundos a sus vidas o, incluso, a su identidad, configurando una nueva normalidad y deben desarrollar nuevas configuraciones en sus relaciones interpersonales y soporte social. Morton, Tong, Howard, Snelling y Webster (2010) completan esta idea en la medida en que se hace importante pensar en las decisiones acerca del tratamiento, pues en la muestra de pacientes con enfermedad renal crónica estudiada por los autores, afirman sentirse limitados en la elección.

Conocer cómo las percepciones varían de acuerdo con los tipos de enfermedades es fuente de datos importantes, a pesar de que los estudios de percepción del riesgo, preocupación, severidad, control en el campo de la salud-enfermedad ya se hayan iniciado hace décadas con la creación de teorías de comportamientos relacionados con las enfermedades (Wanga et al., 2009).

Esta experiencia de vivir como portador de una enfermedad crónica fatal implica estar en constante proceso frente a ciertas inestabilidades del cuadro clínico que redundan en desafíos a través de la subjetividad. 


\section{Relación salud-enfermedad}

En cuanto a la relación salud-enfermedad, la muestra pone en evidencia que la enfermedad es vivida como pérdida de la salud y oscilación entre el momento saludable y el momento enfermo. Esto demuestra que, una vez se es portador de una enfermedad crónica fatal, la persona ya no se siente plenamente saludable. Este hecho refuerza lo muy significativa que es para el paciente la presencia de la enfermedad crónica fatal, ya que cuando ya no pasa desapercibida, deja una marca.

(...) no es nada... la persona enferma no es nada... no es nada de veras... la gente así. (Entrevistado 6)

(...) en el comienzo yo necesité quedarme ingresado... porque yo no lo sabía entonces y pasé mal y comencé a querer hacerme el examen y demás; y tuve que ingresarme... de allá para acá continúo haciendo el tratamiento. ¿Sabes?... Es la vida... no arriesgar, iverdad? (Entrevistado 4)

La dirección de la salud en sí misma es sentida como restrictiva frente a ser portador de una enfermedad incurable. El hecho de no sentirse saludable, cuando se hace posible que solo haya bienestar en lo cotidiano asociado a la libertad de acción que fue limitada por la propia llegada de la enfermedad, son aspectos destacados de esta experiencia.

Los significados que involucran la salud pierden espacio dentro de las normas que la enfermedad instala, propiciando la creación de un universo propio. Vivir saludable no se hace viable dentro del proceso de vivir enfermando, una vez que la enfermedad está ligada con la integridad de la persona.

Bourbeau (2009) apunta hacia la necesidad de buscar la ampliación del abanico de temas que se deben considerar en el tratamiento del portador de Chronic Obstructive Pulmonary Disease (COPD), incluyendo más dimensiones psicológicas, pues estas tienen impacto en las actividades de la vida diaria, afectando su "health status" y su estado funcional limitado. En la misma dirección Balfour, Cooper y Kowal (2006) indican que se deben enfatizar modelos biopsicosociales para mejorar aspectos relacionados con la salud de personas con hepatitis C. Atender los relatos observados en la muestra del presente trabajo, significa identificar los puntos que necesitan ser explorados y las formas de manejarlos mejor.

El automanejo es esencial en el caso de un eficaz cuidado en enfermedades crónicas (McWilliam, 2009), al igual que la importancia de la decisión compartida como medio de facilitar el proceso de tratamiento y facultar a los pacientes (Haslbeck \& Schaeffer, 2011). Dickstein et al. (2008 citado por Smeulders et al., 2010) indican que mejorar habilidades de automanejo en personas con "congestive heart failure" ha sido objeto de creciente atención y puede favorecer los efectos de los síntomas, la capacidad funcional, el bienestar, la morbilidad y el pronóstico. Esta conclusión puede ser aplicada a otras enfermedades crónicas fatales, además de reflexionar sobre la forma en que las intervenciones pueden viabilizar un mayor automanejo de los pacientes en relación con la enfermedad.

Kash, Sharma y Goldfarb (2009) señalan que respecto al manejo de la enfermedad crónica, hay obstáculos tales como inhabilidad de estas personas para adherirse a estilos de vida saludables, falta de congruencia entre las expectativas de proveedores y pacientes, así como también falta de prevención y diagnóstico precoz en muchos cánceres. Según Skott (2002), en pacientes con cáncer se daba una percepción profunda de sí mismos a través de metáforas expresivas. Fue posible percibir el uso de las metáforas en la muestra brasileña como formas de lidiar con el miedo, la ambigüedad y sentimientos íntimos dolorosos al experimentar el proceso de vivir con una enfermedad crónica fatal.

(...) yo me quedo como una planta sin raíz (...) la planta sin raíz no nace de nuevo, ella marchita... ella pierde la vida... es sin raíz...(Entrevistado 7)

(...) esa enfermedad es un fastidio... esa enfermedad es un veneno... es un muerto vivo, ientendiste? (Entrevistado 5)

Sentimientos de falta de vitalidad expresados por los entrevistados involucran un umbral entre lo que está vivo y lo que está muerto. Las carac- 
terísticas de estar vivo no parecen ser suficientes para vivir plenamente, en armonía consigo mismo. La enfermedad parece traer malestares, dependencia física, social, emocional y económica que paulatinamente llevan al proceso de vivir algunas sensaciones incómodas que a veces se aproximan a la muerte o a lo que es considerado como muerto.

Es necesario reflexionar sobre la experiencia de estar en una sociedad post-mortal, donde la muerte es considerada un accidente o una enfermedad que puede ser evitada gracias al control y seguridad de los aparejos técnicos (Lafontaine, 2008 citado por Lafontaine, 2009). En esta perspectiva, no sorprende que la medicina antienvejecimiento y la extensión de la vida capture el imaginario contemporáneo más que cualquier proyecto tecnocientífico (Knorr Cetina, 2005 citado por Lafontaine, 2009).

Así, el temor a la muerte ilustrado en los discursos de los arriba entrevistados, refleja esta sociedad post-mortal que considera la muerte como algo que puede ser impedido. Pero en el caso de la enfermedad crónica fatal, esta cuestión trae un desafío al portador visto que este siente indicios de decrepitud en su cuerpo. A pesar de intentar luchar contra la muerte, siente las dificultades no solamente en el aspecto físico, sino también en los ámbitos psicosociales y económicos.

\section{Consideraciones finales}

La calidad de vida se torna un concepto interesante para ser discutido teniendo en consideración que los portadores de enfermedades crónicas graves apuntan a la posibilidad de cierto confort y bienestar en lo que se refiere a su convivencia con la enfermedad crónica fatal, buscando adaptarse a las condiciones que les son dadas en la sociedad. Hogg et al. (2009) destacan la importancia del trabajo multidisciplinario para mejorar la calidad de los cuidados ofrecidos a los pacientes crónicos y pacientes complejos.

En cuanto al significado del vivir, en los portadores de enfermedades crónicas fatales de la muestra estudiada, la enfermedad parece ocupar, llenar y de alguna manera dar sentido a sus comportamientos y movilizaciones en la vida; esto tal vez traiga una sensación de límite al espacio de la salud o de lo que es saludable. La salud viene siempre asociada al estado de enfermedad.

Sin embargo, este estudio buscó una aproximación a la vivencia de la enfermedad crónica fatal en una muestra brasileña, a través de estudios de casos, y abrió la posibilidad a varias hipótesis para estudios subsecuentes que puedan ampliar y profundizar la temática. Se crearon posibilidades de estudios comparativos cuantitativos-cualitativos entre la experiencia de pacientes portadores de enfermedades crónicas sensibles de control y mantenimiento, en contraste con la experiencia de pacientes de enfermedades crónicas fatales.

La adhesión al tratamiento parece estar relacionada con la lucha por mantenerse vivo; y tratar la enfermedad significa aceptar la existencia en su propio cuerpo y la necesidad de medios medicamentosos o tecnológicos que puedan mantener un bienestar en el día a día.

Los datos analizados y reflexiones de diferentes autores, permiten afirmar que el proceso psicosocial de la experiencia con un cuadro de enfermedad crónica fatal conlleva una crisis no solamente consigo mismo, sino con el entorno social. Pero tales hechos parecen indicar que el mencionado proceso puede derivar en una soledad creativa, que es en parte el resultado de las trabas para mantener concepciones previas de salud y enfermedad, o bien como necesidad de reelaboración de formas de sociabilidad. Por lo tanto, aunque la presencia e influencia de los servicios de salud en la vida de estos enfermos haya sido evidenciada, esto fue acompañado por una valorización de la vida subjetiva individual en el afrontamiento de dichas experiencias extremas de estados físicos. Esto permite que se vuelva a pensar en la asistencia que viene siendo prestada en las unidades de salud, reflexionando sobre el paradigma médico y la forma en que se podrá facilitar el rescate de la personalización o la propia autonomía en ese momento de la vida.

\section{Referencias}

Abric, J. -C. (1994). Pratiques et représentations sociales [Prácticas y representaciones sociales]. París: PUF. 
Balfour, L., Cooper, C. \& Kowal, J. (2006). Depression and cigarette smoking independently relate to reduced health-related quality of life among $\mathrm{Ca}$ nadians living with hepatitis C. Canadian Journal of Gastroenterology, 20(2), 81-86.

Bapat, U., Kedlaya, P. G. \& Gokulnath, A. (2009). Perceived illness intrusion among patients on hemodialisys. Saudi Journal of Kidney Diseases and Transplantation, 20(3), 386-391.

Bardin, L. (1993). El análisis de contenido. Madrid: Ediciones Akal.

Beltrán-Salazar, Ó. A. (2008). La enfermedad grave, una oportunidad. Investigación y Educación en Enfermermería, 26(1), 68-77.

Beltrán-Salazar, Ó. A. (2010). Hay que poner de su parte para afrontar una enfermedad grave. Aquichán, 10(3), 214-227.

Bourbeau, J. (2009). Activities of life: The COPD patient. Journal of Chronic Obstructive Pulmonary Disease, 6(3), 192-200.

Flick, U. (1993). La perception de la santé et la maladie: Théories subjectives et représentations sociales [La percepción de la salud y la enfermedad: teorías subjetivas y representaciones sociales]. París: L'Harmattan.

Flume, P. A., Mogayzel, P. J., Jr., Robinson, K. A., Rosenblatt, R. L., Quittell, L. \& Marshall, B. C. (2010). Cystic fibrosis pulmonary guidelines: Pulmonary complications: Hemoptysis and pneumothorax. American Journal of Respiratory and Critical Care Medicine, 182(39), 298-306.

Frosthlom, L., Fink, P., Christensen, K. S., Toft, T., $\varnothing \mathrm{rnb} \varnothing 1$, E., Olesen, F., et al. (2005). The patients' illness perceptions and the use of primary health care. Psychosomatic Medicine, 67(6), 997-1005.

Giraldo, J. T. (2008). Enfermedad y muerte: un duro camino para vivir. Investigación y Educación en Enfermermería, 26(2), 162-167.

Haslbeck, J. W. \& Schaeffer, D. (2011). Self-responsibility in health care using the example of medication management from the chronically ill patient's perspective. Gesundheitswesen, 73(3), 44-50.

Hogg, W., Lemelin, J., Dahrouge, S., Liddy, C., Deri Armstrong, C., Legault, F., et al. (2009). Randomized controlled trial of anticipatory and preventive multidisciplinary team care for complex patients in a community-based primary care setting. Canadian Family Physician, 55(12), 76-85.

Jeon, Y. H., Kraus, S. G., Jowsey, T. \& Glasgow, N. J. (2010). The experience of living with chronic heart failure: A narrative review of qualitative studies. BMC Health Services Research, 10, 77-86.

Kash, M., Sharma, S. \& Goldfarb, N. I. (2009). Is disease management right for oncology? Population Health Management, 12(6), 337-343.

Lafontaine, C. (2009). Regenerative medicine's immortal body: From the fight against ageing to the extension of longevity. Body Society, 15(4), 53-71.

McWilliam, C. L. (2009). Patients, persons or partners? Involving those with chronic disease in their care. Chronic Illness, 5(4), 277-292.

Morton, R. L., Tong, A., Howard, K., Snelling, P. \& Webster, A. C. (2010). The views of patients and careers in treatment decision making for chronic kidney disease: Systematic review and thematic synthesis of qualitative studies. BMJ, 340, c112. Disponible en http://www.biomedsearch.com/ attachments/00/20/08/59/20085970/bmj.c112.pdf

Moscovici, S. (1961/1976). La psychanalyse, son image et son publique [El psicoanálisis, su imagen y su público]. París: PUF.

Moscovici, S. \& Hewstone, M. (1992). De la science au sens commun [De la ciencia al sentido común]. En S. Moscovici (Ed.), Psychologie sociale (pp. 539566). París: PUF.

Organización Mundial de la Salud. (2010). Innovative care and self-care strategies for people with chronic diseases in Latin America. Revista Panamericana de Salud Pública/Pan American Journal of Public Health, 27(1).

Organización Mundial de la Salud. (2003). Adehence to long-term therapies. Evidence for action. Ginebra: WHO Library Cataloguing-in-Publication Data. Disponible en http://whqlibdoc.who.int/publications/2003/9241545992.pdf

Russell, S. \& Seeley, J. (2010).The transition to living with HIV as a chronic condition in rural Uganda: Working to create order and control when on antiretroviral therapy. Social Science and Medicine, 70(3), 375-82.

Skott, C. (2002). Expressive metaphors in cancer narratives. Cancer Nursing, 25(3), 230-235. 
Smeulders, E. S., van Haastregt, J. C., Ambergen, T., Stoffers, H. E., Janssen-Boyne, J. J., Uszko-Lencer, N. H., et al. (2010). Heart failure patients with a lower educational level and better cognitive status benefit most from a self-management group programme. Patient Education Counseling, 81(2), 214-221.

Wanga, C., O’Neill, S. M., Rothrock, N., Gramling, R., Sen, A., Acheson, L. S., et al. (2009). Comparison of risk perceptions and beliefs across common chronic diseases. Preventive Medicine, 48(2), $197-$ 202.

Zapata, N. E. (2011). La experiencia de sufrir una insuficiencia cardiaca crónica: un padecimiento que acerca a la muerte. Investigación y Educación en Enfermermería, 29(3), 419-426.

Zich, J. \& Temoshok, L. (1987). Perceptions of social support in men with AIDS and ARC: Relationships with distress and hardiness. Journal of Applied Social Psychology, 17(3), 193-215. 
